Article

\title{
Comparative Analysis of the Main Bioactive Components of San-ao Decoction and Its Series of Formulations
}

\author{
Xiaoyun Shu, Yuping Tang *, Chenxue Jiang, Erxing Shang, Xinshen Fan * and Anwei Ding \\ Jiangsu Key Laboratory for High Technology of TCM Formulae Research, \\ Nanjing University of Chinese Medicine, Nanjing 210046, China \\ * Authors to whom correspondence should be addressed; E-Mails: yupingtang@njutcm.edu.cn (Y.T.); \\ fanxsh@njutcm.edu.cn (X.F.); Tel./Fax: +86-25-8581-1916 (Y.T.).
}

Received: 15 October 2012; in revised form: 27 October 2012 / Accepted: 29 October 2012 /

Published: 1 November 2012

\begin{abstract}
A high performance liquid chromatographic (HPLC) method with diode array detection (DAD) was established for simultaneous determination of seven main bioactive components in San-ao decoction and its series of formulae (San-ao decoction, Wu-ao decoction, Qi-ao decoction and Jia-wei San-ao decoction). Seven compounds were analyzed simultaneously with a XTerra $C_{18}$ column $(4.6 \mathrm{~mm} \times 250 \mathrm{~mm}, 5 \mu \mathrm{m})$ using a linear gradient elution of a mobile phase containing acetonitrile (A) and a buffer solution ( $0.02 \mathrm{~mol} / \mathrm{L}$ potassium dihydrogen phosphate and adjusted to $\mathrm{pH} 3$ using phosphoric acid) (B); the flow rate was $1.0 \mathrm{~mL} / \mathrm{min}$. The sample was detected with DAD at 210, 254 and $360 \mathrm{~nm}$ and the column was maintained at $30{ }^{\circ} \mathrm{C}$. All the compounds showed good linearity $\left(r^{2}>0.9984\right)$ in the tested concentration range. The precisions were evaluated by intra-day and inter-day tests, and relative standard deviation (R.S.D.) values within the range of $0.83 \%-2.53 \%$ and $0.64 \%-2.77 \%$ were reported, respectively. The recoveries of the quantified compounds were observed to cover a range from $95.34 \%$ and $104.82 \%$ with R.S.D. values less than $2.72 \%$. The validated method was successfully applied for the simultaneous determination of seven main bioactive components including ephedrine (1), amygdalin (2), liquiritin (3), benzoic acid (4), isoliquiritin (5), formononetin (6) and glycyrrhizic acid (7) in San-ao decoction and its series of formulae. The results also showed a wide variation in the content of the identified active compounds in these samples, which could also be helpful to illustrate the drug interactions after some herbs combined in different formulations.
\end{abstract}


Keywords: San-ao decoction; formulations; Ephedrae Herba; Armeniacae Semen Amarum; Glycyrrhizae Radix et Rhizoma; HPLC-DAD

\section{Introduction}

The composition of Traditional Chinese Medicines (TCMs) is at odds with modern medicine. TCM uses formulae that contain several herbs thought to act in unison to restore what TCM practitioners call the patient's "balance", and some series of formulae (named analogous formulae), groups of formulae with some similarity in medical compositions (combinations) and indications (syndromes), are usually used in TCM clinical practice. Study on series of formulae is an efficient approach to understanding the scientific basis of formulae assembly and syndrome differentiation in TCM, which can also be helpful for elucidating the complex series of Chinese medicine prescriptions and developing new medicines [1-6].

San-ao decoction (SAD) is a representative TCM formula for asthma, which is comprised of three herbs, including Ephedrae Herba (EH), Armeniacae Semen Amarum (AS) and Glycyrrhizae Radix et Rhizoma (GR) at the ratio of 4:10:4. In ancient times, neither the root and eustipes of EH, nor the seed coat of AS were removed, and GR did not need to be processed with honey, thus the formula is not too transpirative or astringent, and was named as "San-ao" decoction. A series of formulae were employed for treatment of asthma diseases in China, of which $42 \%$ were based on SAD, such as Wu-ao decoction (WAD), Qi-ao decoction (QAD) and Jiawei San-ao decoction (JSD) [7]. All of these formulae with their constituent herbs are summarized in Table 1. Nowadays, SAD and its series of formulae are widely used in clinical practice for treating bronchitis caused by bacterial and viral infections. Histopathologic examination showed that SAD suppressed the neutrophil infiltration into lung tissue, which indicated that the antiasthmatic effect of SAD is contributed by its bronchodilator effect and inhibition of neutrophils in the airway [8]. SAD may have a inhibitory effect on airway inflammation of asthmatic rats by regulating Th1/Th2 transfer factors [9]. Our recent research showed that SAD and its series of formulae could alleviate normal human bronchial epithelial cell damage and suppress airway inflammation by various ways [10-18].

Table 1. The composition of San-ao Decoction and its series of formulae.

\begin{tabular}{|c|c|}
\hline Formulae Name & Composition \\
\hline SAD & $\begin{array}{l}\text { Ephedrae Herba (EH, } 4 \text { g), Armeniacae Semen Amarum (AS, } 10 \text { g), } \\
\text { Glycyrrhizae Radix et Rhizoma (GR, } 4 \text { g) }\end{array}$ \\
\hline WAD & $\begin{array}{l}\text { Ephedrae Herba (EH, } 4 \text { g), Armeniacae Semen Amarum (AS, } 10 \text { g), } \\
\text { Glycyrrhizae Radix et Rhizoma (GR, } 4 \text { g), Schizonepetae Herba (SH, } 8 \text { g), } \\
\text { Platycodonis Radix (PR, } 8 \text { g) }\end{array}$ \\
\hline QAD & $\begin{array}{l}\text { Ephedrae Herba (EH, } 4 \text { g), Armeniacae Semen Amarum (AS, } 10 \text { g), } \\
\text { Glycyrrhizae Radix et Rhizoma (GR, } 4 \text { g), Pinelliae Rhizoma (PR, } 10 \text { g), } \\
\text { Schisandrae Chinensis Fructus (SC, } 6 \text { g), Gypsum Fibrosum (GF, } 20 \text { g), } \\
\text { Camelliae sinensis Folium (CS, } 10 \text { g) }\end{array}$ \\
\hline JSD & $\begin{array}{l}\text { Ephedrae Herba (EH, } 4 \mathrm{~g} \text { ), Armeniacae Semen Amarum (AS, } 10 \mathrm{~g} \text { ), } \\
\text { Glycyrrhizae Radix et Rhizoma (GR, } 4 \text { g), Asari Radix et Rhizoma (AR, } 10 \mathrm{~g} \text { ) }\end{array}$ \\
\hline
\end{tabular}


Ephedrine, which is the bio-marker constituent in EH, can relax bronchial smooth muscle [19]. Amygdalin had a sedative effect on the respiratory center [20], and benzoic acid could improve the anticancer effects [21]. Glycyrrhizic acid could suppresses Cox-2-mediated anti-inflammatory responses [22]; liquiritin had antidepressant effects [23]; isoliquiritin had antigenotoxic effects [24]; formononetin could reduce production of proinflammatry mediators in LPS-stimulated macrophages, fibroblasts, and intestinal epithelial cells [14]. It was reported that these constituents were the main active compounds of SAD $[13,14]$. To date, some preliminary research concerning the quantitative analysis of active components in SAD using HPLC methods have been reported [25-30]. However, there is little information available in the literature about the variation of the main bio-active constituents in San-ao decoction and its series of formulae, therefore, it is necessary to establish a method for comparative characterization of these active components in these formulae, which is also useful to illustrate the changes of the contents of these marker compounds after herbs are combined into different formulae.

In recent years, some researchers have started to become interested in the compatibility rules of the TCM formulae by comparatively analyzing some of the main bioactive components of different herbs or formulae [31-37]. This study aimed to develop a direct and convenient high performance liquid chromatographic coupled with diode array detector (HPLC-DAD) method to simultaneously analyze quantitatively the seven main bio-active components in San-ao decoction and its series of formulae, including ephedrine (1), amygdalin (2), liquiritin (3), benzoic acid (4), isoliquiritin (5), formononetin (6) and glycyrrhizic acid (7) (Figure 1). The study will lay a foundation to establish the quality standards for these formulae and the results were also helpful to illustrate the drug interactions after some herbs are combined into different formulae.

Figure 1. The structures of the reference compounds: ephedrine (1), amygdalin (2), liquiritin (3), benzoic acid (4), isoliquiritin (5), formononetin (6) and glycyrrhizic acid (7).

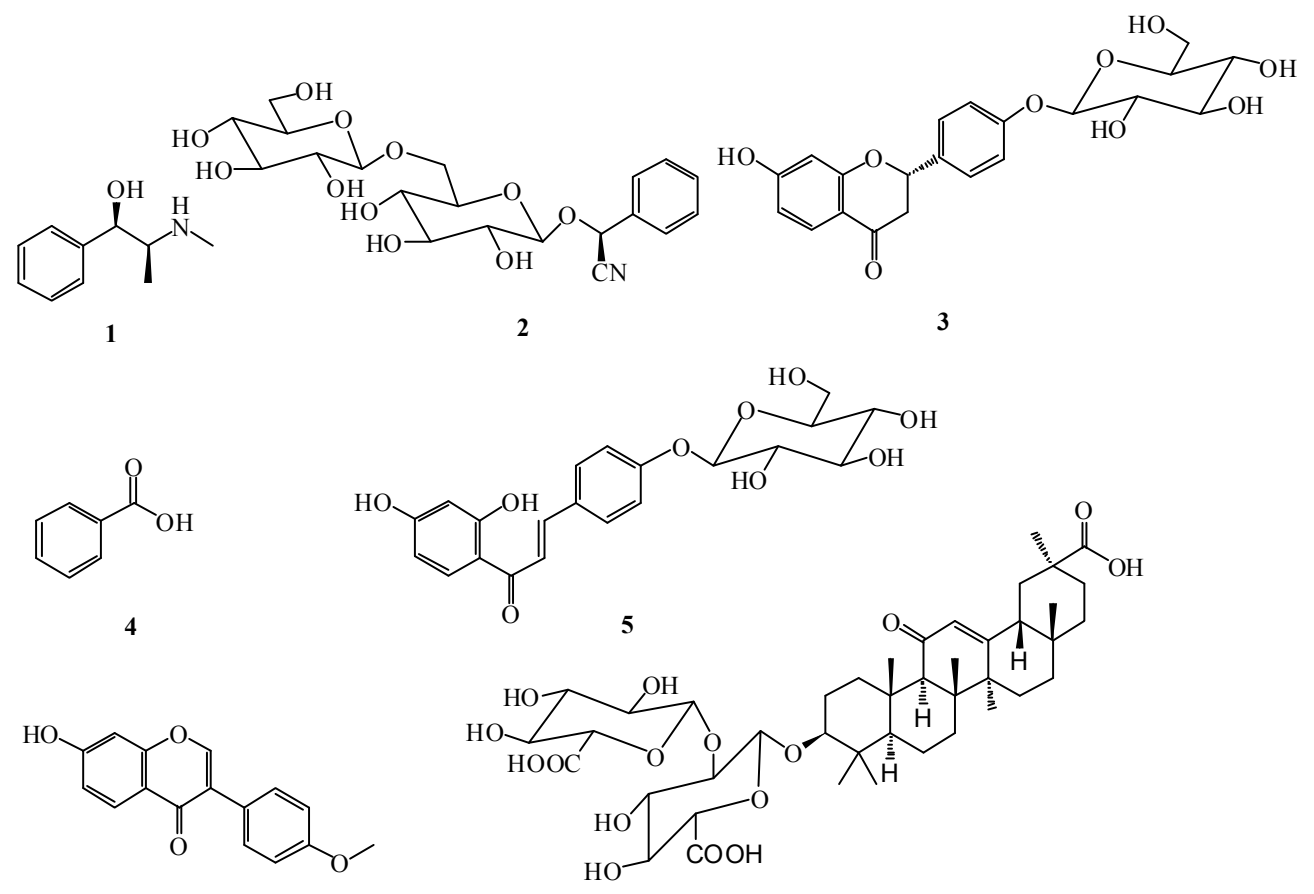




\section{Results and Discussion}

\subsection{Method Validation}

The chromatographic methods were validated to determine the linearity, LOD, LOQ, intraday and interday precisions, and accuracy. A stock solution containing the seven reference compounds was prepared and diluted to seven appropriate concentrations to obtain the calibration curves, which were plotted after linear regression of the peak areas compared with the corresponding concentrations. The lowest concentration of working solution was diluted with methanol to a series of appropriate concentrations, and aliquots of these diluted solutions were injected into the HPLC for analysis. The LOD was determined at a signal-to-noise $(\mathrm{S} / \mathrm{N})$ ratio of 3, and LOQ was determined at a $\mathrm{S} / \mathrm{N}$ ratio of 10. The calibration curve results indicated that all seven reference compounds showed good linearity $\left(r^{2}>0.9984\right)$ within relatively wide concentration ranges. The LODs and LOQs of the seven analytes were $0.10-3.70$ and $0.60-11.10 \mathrm{ng}$, respectively. The linearity, LOD and LOQ values of each analyte are presented in Table 2.

Table 2. Regression equation, correlation coefficients, linearity ranges, limits of detection (LOD) and limits of quantification (LOQ) of investigated compounds.

\begin{tabular}{llcccc}
\hline \multicolumn{1}{c}{ Analytes } & \multicolumn{1}{c}{ Regression equation } & $\boldsymbol{r}^{2}$ & $\begin{array}{c}\text { Linear range } \\
(\boldsymbol{\mu g})\end{array}$ & $\begin{array}{c}\text { LOD }^{\mathrm{b}} \\
(\mathbf{n g})\end{array}$ & $\begin{array}{c}\mathbf{L O Q}^{\mathrm{c}} \\
(\mathbf{n g})\end{array}$ \\
\hline Ephedrine & ${ }^{\mathrm{a}} \mathrm{y}=600818 \mathrm{x}+78730$ & 0.9997 & $0.32 \sim 20.56$ & 1.60 & 4.80 \\
Amygdalin & $\mathrm{y}=469675 \mathrm{x}-4930$ & 0.9986 & $0.74 \sim 47.60$ & 3.70 & 11.10 \\
Liquiritin & $\mathrm{y}=385254 \mathrm{x}+5938$ & 0.9984 & $0.16 \sim 10.40$ & 0.80 & 2.40 \\
Benzoic acid & $\mathrm{y}=5493640 \mathrm{x}-27349$ & 0.9998 & $0.02 \sim 1.12$ & 0.10 & 0.30 \\
Isoliquiritin & $\mathrm{y}=6553375 \mathrm{x}-227224$ & 0.9994 & $0.10 \sim 6.16$ & 0.50 & 1.50 \\
Formononetin & $\mathrm{y}=888962 \mathrm{x}+22087$ & 0.9984 & $0.04 \sim 2.56$ & 0.20 & 0.60 \\
Glycyrrhizic acid & $\mathrm{y}=803987 \mathrm{x}-134817$ & 0.9987 & $0.43 \sim 27.44$ & 2.15 & 6.45 \\
\hline
\end{tabular}

${ }^{\mathrm{a}} \mathrm{y}$ is the peak area in UV chromatograms monitored at the absorption maximum for each reference compound, $\mathrm{x}$ is the compound amount injected (ug). ${ }^{\mathrm{b}}$ LOD refers to the limits of detection. ${ }^{\mathrm{c}}$ LOQ refers to the limits of quantification.

The intraday and interday precisions were investigated by analyzing known concentrations of analytes in six replicates during a single day and by duplicating the experiments on three successive days, respectively. The relative standard deviation (RSD) was taken as a measure of precision. The results indicated that the intra- and interday RSD values of the seven compounds were all lower than $3.0 \%$ (Table 3 ). The repeatability was assessed by analyzing six independently prepared samples using the same method. Sample stability was evaluated at room temperature and analyzed at $0,2,4,8,12$ and $24 \mathrm{~h}$ within one day, respectively. The RSD was taken as a measure of repeatability and stability and the results of the seven compounds in SAD are showed in Table 3. Recovery was calculated by spiking accurate amounts of the seven standards at low (80\% of the known amounts), medium (same as the known amounts), and high (120\% of the known amounts) to SAD sample. The resultant samples were then extracted and analyzed by using the proposed procedure. As shown in Table 3, the recoveries of the seven compounds were in the range of $95.34 \%-104.82 \%$, which indicated that the 
methods were accurate enough for the determination of these compounds in SAD and its series of formulae.

Table 3. Precision, repeatability stability and recovery of the seven analytes.

\begin{tabular}{|c|c|c|c|c|c|c|}
\hline \multirow[b]{2}{*}{ Analytes } & \multicolumn{2}{|c|}{ Precision (R.S.D., \%) } & \multirow{2}{*}{$\begin{array}{l}\text { Repetability } \\
\text { (R.S.D., \%, } \\
\quad n=6 \text { ) }\end{array}$} & \multirow{2}{*}{$\begin{array}{c}\text { Stability } \\
\text { (R.S.D., \%, } \\
n=6)\end{array}$} & \multicolumn{2}{|c|}{ Recovery $(\%, n=9)$} \\
\hline & $\begin{array}{c}\text { Intra-day } \\
(n=6)\end{array}$ & $\begin{array}{c}\text { Inter-day } \\
\quad(n=3)\end{array}$ & & & Mean & R.S.D. (\%) \\
\hline Ephedrine & 1.21 & 1.69 & 0.56 & 1.82 & 103.13 & 2.26 \\
\hline Amygdalin & 1.84 & 2.77 & 1.72 & 2.13 & 104.82 & 2.72 \\
\hline Liquiritin & 0.93 & 0.64 & 1.22 & 1.85 & 96.27 & 1.55 \\
\hline Benzoic acid & 2.53 & 2.19 & 1.84 & 1.32 & 97.14 & 2.69 \\
\hline Isoliquiritin & 1.79 & 1.94 & 1.84 & 2.04 & 96.26 & 1.41 \\
\hline Formononetin & 1.22 & 1.73 & 0.95 & 2.17 & 95.34 & 2.38 \\
\hline Glycyrrhizic acid & 0.83 & 0.69 & 0.91 & 2.59 & 98.39 & 1.16 \\
\hline
\end{tabular}

\subsection{Sample Analysis}

The established analytical method was then subsequently applied to a simultaneous determination of the seven markers of SAD and its series of formulae. Typical HPLC chromatograms of mixed standards and the investigated samples at 210, 254 and $360 \mathrm{~nm}$ are shown in Figures 2-4, respectively. The results (Table 4) showed that the content of the seven constituents displayed striking differences among the analyzed samples. The contents variation of compounds 1-7 were as follows: for compound 1: $\mathrm{QAD}>\mathrm{JSD}>\mathrm{SAD}>\mathrm{WAD}$; for compounds 2, 3 and 5: $\mathrm{QAD}>\mathrm{WAD}>\mathrm{SAD}>\mathrm{JSD}$; for compound 4: $\mathrm{WAD}>\mathrm{SAD}>\mathrm{JSD}>\mathrm{QAD}$; for compounds 6 and 7: $\mathrm{SAD}>\mathrm{WAD}>\mathrm{JSD}>\mathrm{QAD}$. In comparison with SAD, the contents of compounds 2-5 and 7 in WAD were higher than those in SAD, but the contents of ephedrine and formononetin decreased after SH and PR were added into SAD; the contents of compounds 1-3 in QAD were higher than those in SAD, and other compounds decreased after PR, $\mathrm{SC}, \mathrm{GF}, \mathrm{CS}$ were added into SAD; the contents of compounds 2-7 in JSD were lower than those in SAD, but the contents of ephedrine increased after AR was added into SAD. And the total content of the seven bio-active constituents in QAD was highest in the four TCM formulae, which supported the interpretation that QAD had the best therapeutic effect on asthmatic mice induced by ovalbumin sensitization and RSV infection $[11,12]$.

Figure 2. Typical HPLC chromatograms of mixed standards and the investigated samples at $210 \mathrm{~nm}$. ephedrine (1), amygdalin (2), liquiritin (3), benzoic acid (4), isoliquiritin (5), formononetin (6) and glycyrrhizic acid (7).

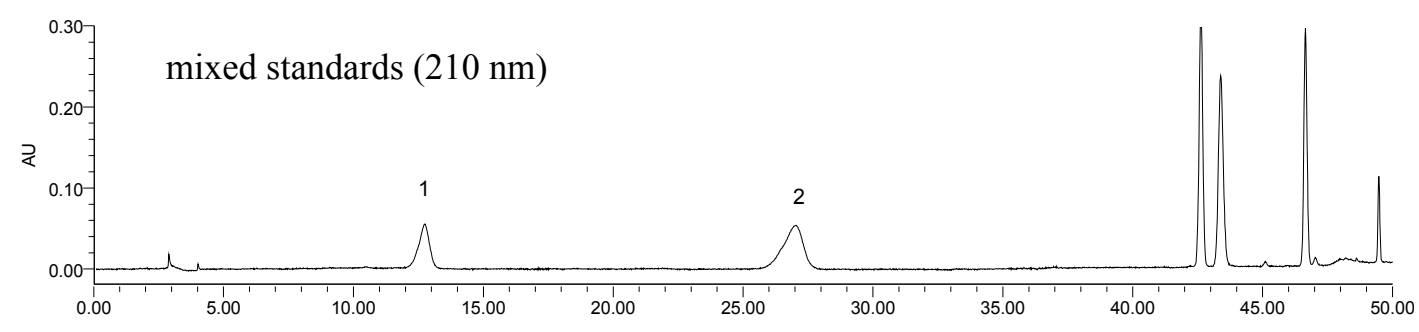


Figure 2. Cont.
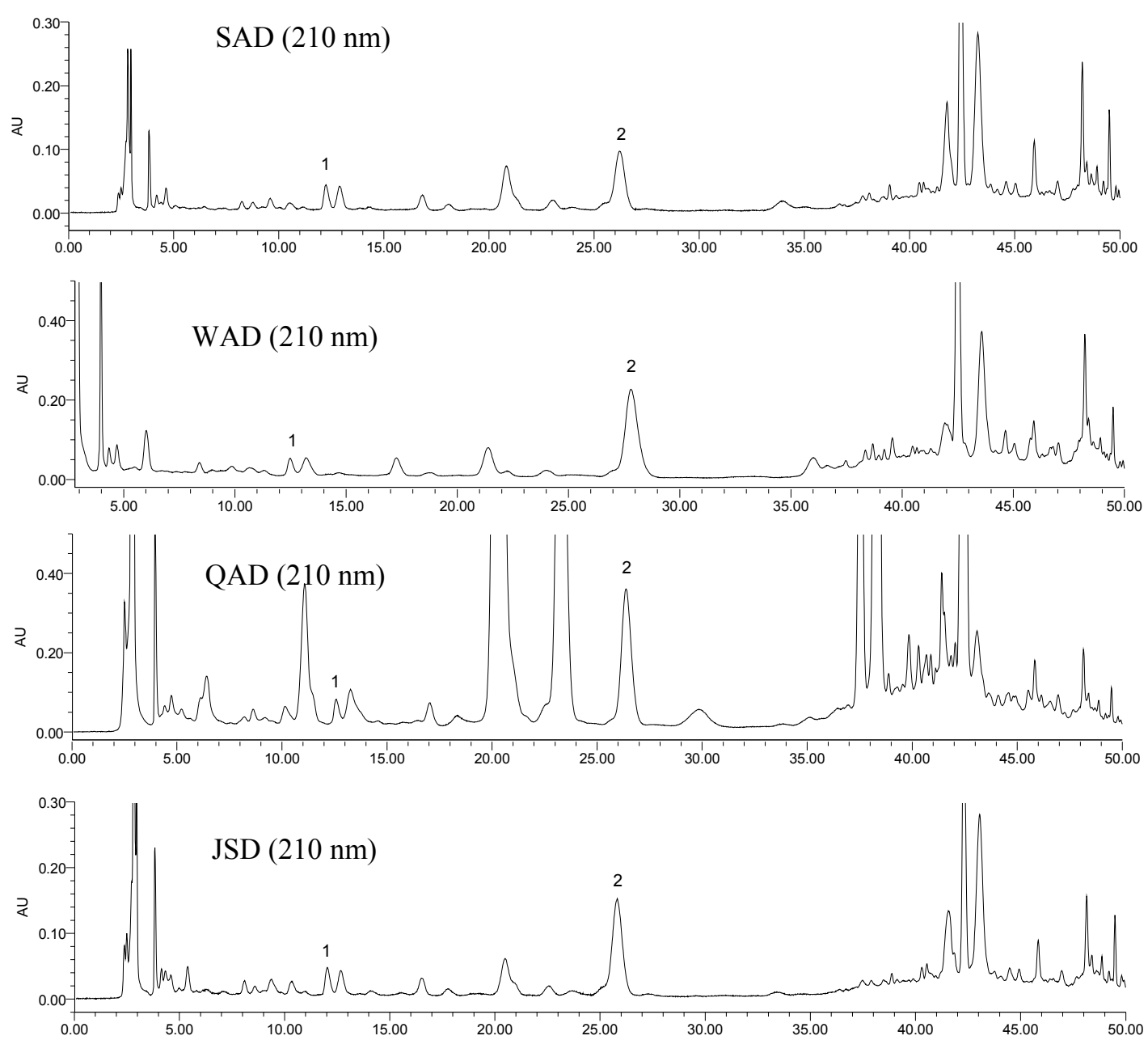

Figure 3. Typical HPLC chromatograms of mixed standards and the investigated samples at $254 \mathrm{~nm}$. ephedrine (1), amygdalin (2), liquiritin (3), benzoic acid (4), isoliquiritin (5), formononetin (6) and glycyrrhizic acid (7).
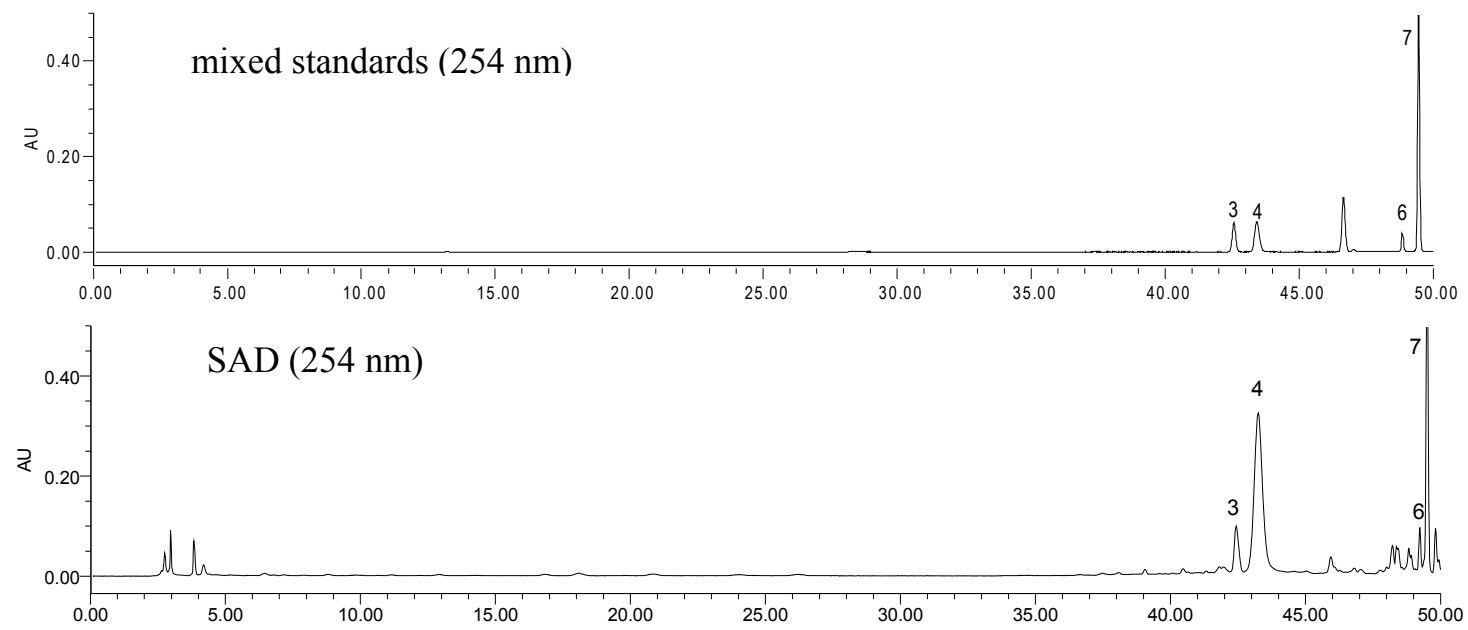
Figure 3. Cont.
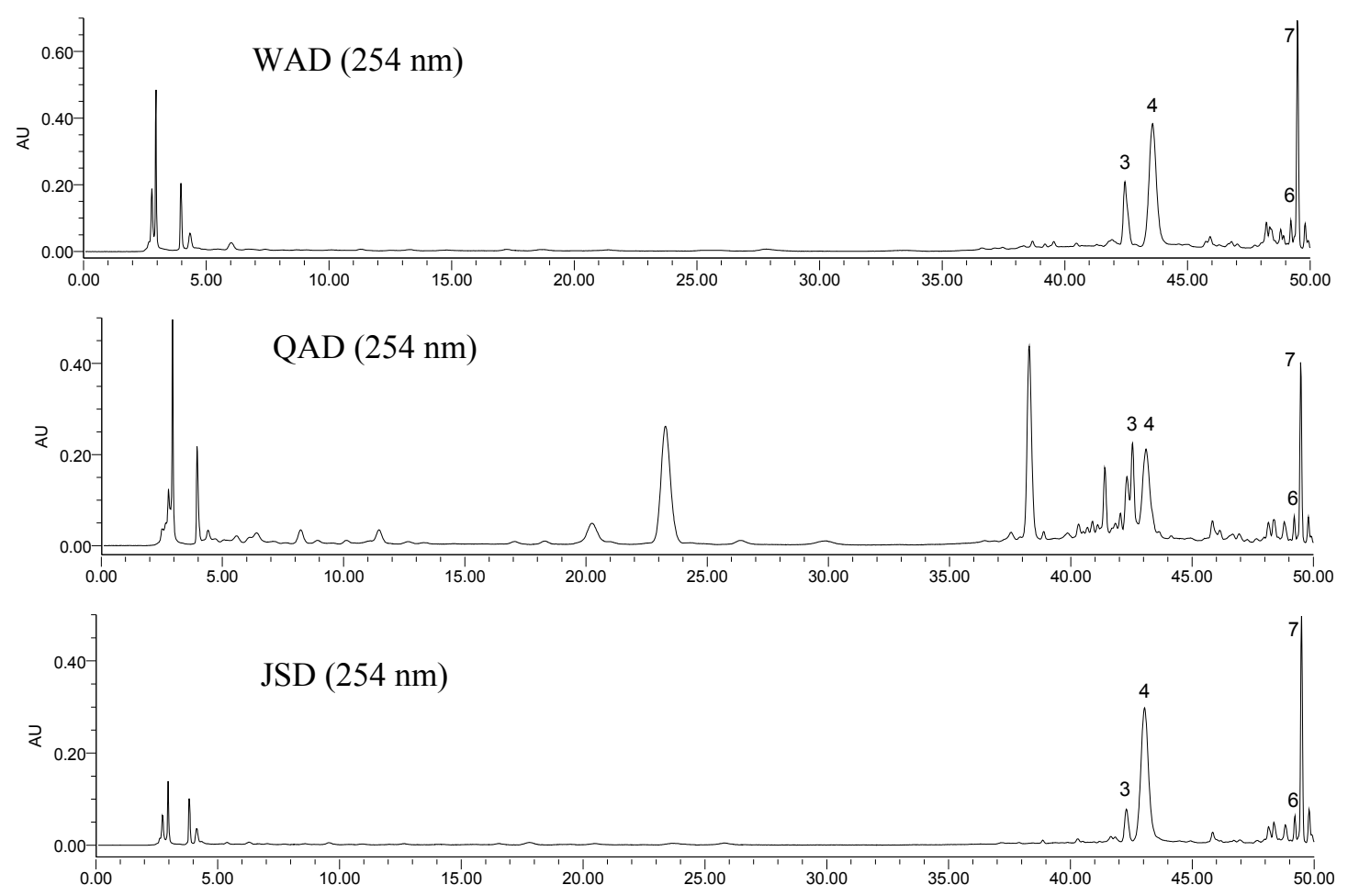

Figure 4. Typical HPLC chromatograms of mixed standards and the investigated samples at $360 \mathrm{~nm}$. ephedrine (1), amygdalin (2), liquiritin (3), benzoic acid (4), isoliquiritin (5), formononetin (6) and glycyrrhizic acid (7).
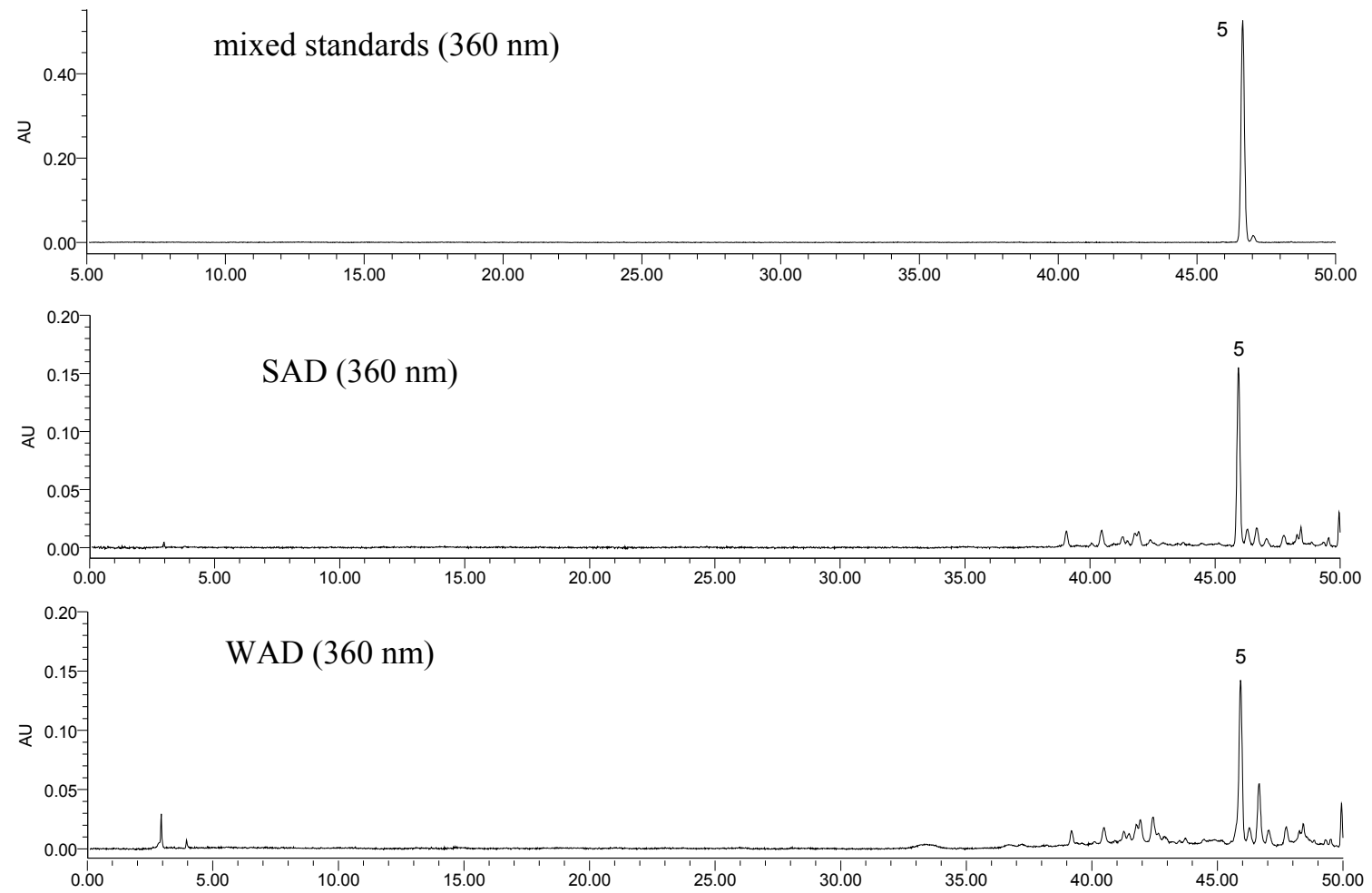
Figure 4. Cont.
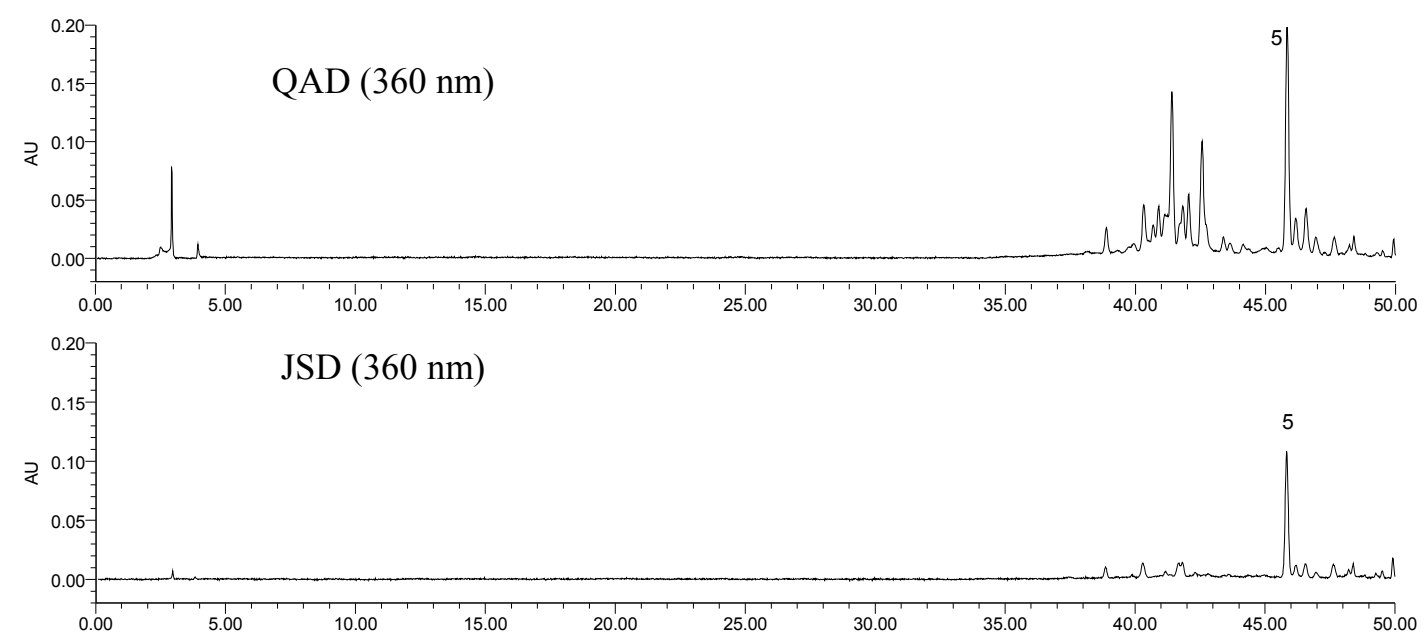

Table 4. Contents of the seven studied compounds in the investigated samples $(n=3)$.

\begin{tabular}{ccccccccc}
\hline \multirow{2}{*}{ Sample No. } & $\mathbf{7}$ Content (mg/g) & \multirow{2}{*}{ Total } \\
\cline { 2 - 7 } & $\mathbf{1}$ & $\mathbf{2}$ & $\mathbf{3}$ & $\mathbf{4}$ & $\mathbf{5}$ & $\mathbf{6}$ & $\mathbf{7}$ & \\
\hline SAD & 2.24 & 13.28 & 6.79 & 1.25 & 0.59 & 1.23 & 12.18 & 37.56 \\
WAD & 2.23 & 19.12 & 11.69 & 1.41 & 0.61 & 1.05 & 12.41 & 48.52 \\
QAD & 2.99 & 24.82 & 12.77 & 0.71 & 0.75 & 0.7 & 7.19 & 49.93 \\
JSD & 2.39 & 10.69 & 4.85 & 1.08 & 0.42 & 0.72 & 8.66 & 28.81 \\
\hline
\end{tabular}

These results demonstrated that $\mathrm{SH}$ and $\mathrm{PR}$ could significantly promote the dissolution of amygdalin (2) in AS and liquiritin (3) in GR during their preparation procedure; PR, SC, GF and CS could greatly improve the dissolution of ephedrine (1) in EH, amygdalin (2) in AS and liquiritin (3) in GR, and obviously reduce the dissolution of benzoic acid (4) in AS, formononetin (6) and glycyrrhizic acid (7) in GR; AR might obviously reduce the dissolution of formononetin (6) and glycyrrhizic acid (7) in GR. The results also suggested that some drug-drug interactions exist, such as depression and enhancement of extractive rate of the bioactive components in SAD and its series of formulae during their preparation procedure. Thus, when SAD and its series of formulae are used for disease treatment, the relative proportions of the herbs should be fixed to avoid any changes of bioactive component contents.

\section{Experimental}

\subsection{Plant Materials}

Ephedrae Herba (the dried herbaceous stem and root of Ephedra sinica Stapf) was collected from Xinjiang Province; Armeniacae Semen Amarum (the dried ripe seed with coat of Prunus armeniaca L. var. ansu Maxim.) and Gypsum Fibrosum $\left(\mathrm{GaSO}_{4} \cdot 2 \mathrm{H}_{2} \mathrm{O}\right)$ were collected from Shandong Province; Glycyrrhizae Radix et Rhizoma (the dried root and rhizome of Glycyrrhiza uralensis Fisch.) was collected from Ningxia Province; Schizonepetae Herba (Schizonepeta tenuifolia Briq.) and Platycodonis Radix (Platycodon grandiflorum (Jacq.) A. DC.) were collected from Anhui Province; Pinelliae Rhizoma (Pinellia ternate (Thunb.) Breit.) was collected from Sichuan Province; Camelliae sinensis Folium (Camellia sinensis (L.) O. Kuntze) was collected from Jiangsu Province; Schisandrae 
Chinensis Fructus (Schisandra chinensis (Turcz.) Baill.) and Asari Radix et Rhizoma (Asarum heterotropoides Fr. Schmidt var. mandshuricum (Maxim.) Kitag) were collected from Liaoning Province. The crude herbs were identified by Professor Dekang Wu (Department of Pharmacognosy, Nanjing University of Chinese Medicine), and voucher specimens were deposited in the Herbarium of Nanjing University of Chinese Medicine, China.

\subsection{Chemicals and Reagents}

The reference standards of ephedrine, amygdalin, liquiritin and formononetin were obtained from National Institute for the Control of Pharmaceutical and Biological Products of China (Beijing, China). Isoliquiritin and glycyrrhizic acid were purchased from Shanghai Winherb Medical Science Co., Limited (Shanghai, China). Benzoic acid was provided by our laboratory and its structure was determined by ${ }^{1} \mathrm{H}-\mathrm{NMR},{ }^{13} \mathrm{C}-\mathrm{NMR}$, MS and UV spectra (purity $>98 \%$ ). The chemical structures of these reference compounds are shown in Figure 1. Acetonitrile (HPLC grade) and methanol (HPLC grade) were obtained from Tedia Inc. (Fairfield, OH, USA). EtOAc and ethanol were obtained from Hanbang Company (Huaian, China). Unless specified elsewhere, all reagents were used without further purification. Distilled and deionized water was purified by the superpure water system (Eped Technology Development Co., Ltd., Nanjing, China).

\subsection{Preparation of Standard Solutions}

A mixed standard stock solution containing ephedrine (1), amygdalin (2), liquiritin (3), benzoic acid (4), isoliquiritin (5), formononetin (6) and glycyrrhizic acid (7) was prepared in methanol. Working standard solutions were prepared by diluting the mixed standard stock solution with methanol to give seven different quantities within the ranges: $0.32-20.56 \mu \mathrm{g} ; 0.74-47.60 \mu \mathrm{g}$; 0.16-10.40 $\mu \mathrm{g}$; 0.02-1.12 $\mu \mathrm{g}$; $0.10-6.16 \mu \mathrm{g} ; 0.04-2.56 \mu \mathrm{g}$ and $0.43-27.44 \mu \mathrm{g}$ for calibration curves. The standard solutions were filtered through a $0.45 \mu \mathrm{m}$ membrane prior to injection. All solutions were stored in a refrigerator at $4{ }^{\circ} \mathrm{C}$ before analysis.

\subsection{Sample Preparation}

The preparation of $S A D: 4 \mathrm{~g}$ of $\mathrm{EH}, 10 \mathrm{~g}$ of $\mathrm{AS}$ and $4 \mathrm{~g}$ of GR were weighed in proportion and decocted together with boiling water twice for $2 \mathrm{~h}$ each time. The water extract solution was combined and the solvent was removed under vacuum below $65^{\circ} \mathrm{C}$ till a certain volume at the ratio of $1: 1(\mathrm{w} / \mathrm{w}$, weight of all herbs and the extracted filtrates) was reached, and then ethanol was added slowly with churning all the time until the ethanol content reached $80 \%$. After standing for $24 \mathrm{~h}$, the solution was filtered to remove the deposits and concentrated to a certain concentration under vacuum below $65{ }^{\circ} \mathrm{C}$, and then partitioned with EtOAc. The EtOAc fraction was accurately weighed and dissolved in $10 \mathrm{~mL}$ of $80 \%$ methanol; sonicated twice for 30 min each time with a short interval; the extracted solution was diluted five times with methanol; afterwards, it was filtered through a $0.45 \mu \mathrm{m}$ filter membrane. The filtrate was used as the sample solution of SAD. The preparation of WAD: $4 \mathrm{~g}$ of EH, $10 \mathrm{~g}$ of AS, $4 \mathrm{~g}$ of GR, $8 \mathrm{~g}$ of SH and $8 \mathrm{~g}$ of PR were weighed and prepared in the same way as SAD. The preparation of $Q A D: 4 \mathrm{~g}$ of EH, $10 \mathrm{~g}$ of AS, $4 \mathrm{~g}$ of GR, $10 \mathrm{~g}$ of PR, $6 \mathrm{~g}$ of SC, $20 \mathrm{~g}$ of GF and $10 \mathrm{~g}$ of CS were 
weighed and prepared in the same way as SAD. The preparation of JSD: $4 \mathrm{~g}$ of EH, $10 \mathrm{~g}$ of AS, $4 \mathrm{~g}$ of $\mathrm{GR}$, and $3 \mathrm{~g}$ of $\mathrm{AR}$ were weighed and prepared in the same way as SAD.

\subsection{Apparatus and Chromatographic Conditions}

Analysis was performed on a Waters 2695 HPLC system, equipped with a binary pump, an autosampler and a column oven coupled to a variable wavelength DAD (Waters Corp., Milford, MA, USA). HPLC separation of terpenoids was achieved using a XTerra $\mathrm{C}_{18}$ column $(4.6 \times 250 \mathrm{~mm}$, $5.0 \mu \mathrm{m}$, Waters). The separation of seven compounds was achieved by a linear gradient elution of a mobile phase containing acetonitrile (A) and a buffer solution $(0.02 \mathrm{~mol} / \mathrm{L}$ potassium dihydrogen phosphate and adjusted $\mathrm{pH} 3$ using phosphoric acid) (B). The gradient profile was as follows: 0-15 min: linear 5\%-8\% of $\mathrm{A}(\mathrm{v} / \mathrm{v}) ; 15-30 \mathrm{~min}$ : isocratic $8 \%$ of $\mathrm{A} ; 30-44 \mathrm{~min}: 8 \%-32 \% \mathrm{~A}$; 44-50 min: $32 \%-95 \%$ of A. A re-equilibration period of 10 min was used between individual runs. Detection wavelength was set at $210 \mathrm{~nm}$ for reference compounds $1-2 ; 254 \mathrm{~nm}$ for 3, 4, 6, 7 and $360 \mathrm{~nm}$ for 5 . Chromatography was carried out at $30{ }^{\circ} \mathrm{C}$ with a flow rate of $1.0 \mathrm{~mL} / \mathrm{min}$. An injection volume of $10 \mu \mathrm{L}$ was used.

\subsection{Validation of the Methods}

The dilute solution of the reference compounds was further diluted to a series of concentrations with methanol to assess the limits of detection (LOD) and quantification (LOQ). The LOD and LOQ were determined at signal-to-noise $(\mathrm{S} / \mathrm{N})$ ratios of 3 and 10 , respectively. The intraday and interday precision was determined by analyzing calibration samples during a single day and on three consecutive days, respectively. To confirm the repeatability, six different working solutions were analyzed. The R.S.D. was taken as a measure of precision and reproducibility. A recovery test was used to evaluate the accuracy of the method. In the test, reference compounds were added to SAD at low (80\% of the known amounts), medium (same as the known amounts) and high (120\% of the known amounts) levels.

\section{Conclusions}

In the present study, chromatograms for determination of the seven major bio-active components in SAD and its series of formulae were developed and applied by using an HPLC-DAD method. Method validation of linearity, precision, repeatability, stability and accuracy were acceptable. The results demonstrated that the proposed method was able to detect comprehensive content changes after the relative herbs were combined into San-ao decoction or its series of formulae. The research also provided some experimental reference data for clinic applications of these formulae.

\section{Acknowledgments}

This research was financially supported by National Natural Science Foundation of China (81072748), National 11th Five-Year Scientific and Technological Support Projects (2006BAI06A02-01), 2009' Program for New Century Excellent Talents by the Ministry of Education (NCET-09-0163). This research was also financially supported by Construction Project for Jiangsu Engineering Center of 
Innovative Drug from Blood-conditioning TCM Formulae, and A Project Funded by the Priority Academic Program Development of Jiangsu Higher Education Institutions (ysxk-2010). We are also pleased to thank Waters China Ltd. for technical support.

\section{References}

1. Fan, X.S.; Duan, J.A.; Ding, A.W. Four issues of basic research on categorized formulae of Chinese medicine. Zhongguo Zhongxiyi Jiehe Zazhi 2010, 37, 246-251.

2. Liu, L.; Ma, H.Y.; Tang, Y.P.; Chen, W.X.; Lu, Y.; Guo, J.M.; Duan, J.A. Discovery of estrogen receptor a modulators from natural compounds in $\mathrm{Si}-\mathrm{Wu}$-Tang series decoctions using estrogenresponsive MCF-7 breast cancer cells. Bioorg. Med. Chem. Lett. 2012, 22, 154-163.

3. Zhu, M.; Tang, Y.P.; Duan, J.A.; Guo, J.M.; Guo, S.; Su, S.L.; Shang, E.X.; Qian, D.W.; Ding, A.W. Roles of paeoniflorin and senkyunolide I in SiWu decoction on antiplatelet and anticoagulation activities. J. Sep. Sci. 2010, 33, 3335-3340.

4. Liu, L.; Duan, J.A.; Tang, Y.P.; Ma, H.Y.; Su, S.L.; Guo, J.M.; Hua, Y.Q. The protective effects of the active fraction of Shaofu Zhuyu decoction on hydrogen peroxide-induced oxidative injury in vascular smooth muscle cells. Molecules 2010, 15, 5066-5078.

5. Liu, P.; Duan, J.A.; Hua, Y.Q.; Tang, Y.P.; Yao, X.; Su, S.L. Effects of Xiang-Fu-Si-Wu Decoction and its main components for dysmenorrhea on uterus contraction. J. Ethnopharmacol. 2011, 133, 591-597.

6. Su, S.L.; Yu, L.; Hua, Y.Q.; Duan, J.A.; Deng, H.S.; Tang, Y.P.; Lu, Y.; Ding, A.W. Screening and analysis of potential bioactive components from Shaofu Zhuyu decoction, using human umbilical vein endothelial cell extract and high performance liquid chromatography coupled with Q-TOF/MS spectrometry. Biomed. Chromatogr. 2008, 22, 1385-1392.

7. Fan, X.S. Discussion on application of San'ao decoction and its analogous prescription. Anhui Zhongyi Xuebao 2006, 25, 1-3.

8. Kao, S.T.; Yeh, T.J.; Hsieh, C.C.; Yeh, F.T.; Lin, J.G. Effect of San-Ao-Tang on immediate and late airway response and leukocyte infiltration in asthmatic guinea pigs. Immunopharmacol. Immunotoxicol. 2000, 22, 143-162.

9. Chen, H.; Ma, R. Study on Th1/Th2 transcriptional regulatory mechanism of san'ao tang in bronchial asthmatic rats. Zhongguo Zhongyao Zazhi 2012, 37, 1324-1326.

10. Gu, P.C.; Fan, X.S.; Jiang, C.X.; Xu, H.Q.; Yu, J.H.; Tang, Y.P. Effect of San'ao Decoction on the airway inflammation and hyperresponsiveness in a murine model of lipopolysaccharide-enhanced asthma. Chin. J. Integr. Med. 2011, 17, 537-541.

11. Xu, H.Q.; Gu, P.C.; Fan, X.S.; Tong, H.G.; Zhang, Y.; Chen, J.D. Effect of San-ao decoction and its analogous prescription on airway responsiveness in asthmatic mice induced by ovalbumin sensitisation and RSV infection. Shijie Kexue Jishu-Zhongyiyao Xiandaihua 2009, 11, 702-706.

12. Zhang, Y.; Tong, H.J.; Yu, J.H.; Gu, P.C.; Fan, X.S.; Xu, H.Q. Effects of San-ao decoction and its analogous prescriptions on airway inflammation in mice with respiratory syncytial virus-and ovalbumin-induced asthma. Zhongxiyi Jiehe Xuebao 2009, 7, 354-359. 
13. Zhou, L.; Tang, Y.P.; Liu, C.M.; Gao, L.; Fan, X.S.; Wu, D.K.; Duan, J.A. Chemical constituents from San-ao decoction and their effects on the activation of PPAR $\gamma$. Asian Chem. Lett. 2009, 13, $47-52$.

14. Zhou, L.; Tang, Y.P.; Gao, L.; Fan, X.S.; Liu, C.M.; Wu, D.K. Separation, characterization and dose-effect relationship of the PPARgamma-activating bio-active constituents in the Chinese herb formulation "San-Ao decoction". Molecules 2009, 14, 3942-3951.

15. Wang, S.S.; Feng, L.; Xu, L.; Shi, L.; Fan, X.S. Effects of San'ao decoction and its modification on airway inflammation of TMA-invoked rat asthma model. Nanjing Zhongyiyao Daxue Xuebao 2011, 27, 542-545.

16. Ma, C.H.; Wang, S.S.; Ma, S.P.; Fan, X.S. Effects of San'ao decoction on allergic airway inflammation in asthma mouse and its component analysis. Zhongguo Shiyan Fangjixue Zazhi 2012, 18, 139-143.

17. Li, Y.; Wang, M.Y.; Fan, X.S.; Qi, X.; Chen, Y.; Zhang, H.; Zhao, Q.C. Effect of San-ao Decoction, a traditional Chinese prescription, on IL-4 treated normal human bronchial epithelium. J. Ethnopharmacol. 2010, 131, 104-109.

18. Wang, S.S.; Fan, X.S; Xu, L.;Yu, J.H.; Ma, C.H. Study on the compatibility of Sanao tang based on orthogonal experiment. Zhongguo Shiyan Fangjixue Zazhi 2012, 18, 149-153.

19. Yuan, D.; Komatsu, K.; Tani, H.; Cui, Z.; Kano, Y. Pharmacological properties of traditional medicines. XXIV. Classification of antiasthmatics based on constitutional predispositions. Biol. Pharm. Bull. 1998, 21, 1169-1173.

20. Bak, I.; Lekli, I.; Juhasz, B.; Varga, E.; Varga, B.; Gesztelyi, R.; Szendrei, L.; Tosaki, A. Isolation and analysis of bioactive constituents of sour cherry (Prunus cerasus) seed kernel: An emerging functional food. J. Med. Food 2010, 13, 905-910.

21. Wang, M.; Qin, L.; Li, K.; Zhu, R.; Wang, W.; Wang, S. The improvement of the anticancer effect of a novel compound benzoic acid, 2-hydroxy-, 2-D-ribofuranosylhydrazide (BHR) loaded in solid lipid nanoparticles. AAPS PharmSciTech 2012, doi:10.1208/s12249-012-9862-8.

22. Bhattacharjee, S.; Bhattacharjee, A.; Majumder, S.; Majumdar, S.B.; Majumdar, S. Glycyrrhizic acid suppresses Cox-2-mediated anti-inflammatory responses during Leishmania donovani infection. J. Antimicrob. Chemother. 2012, 67, 1905-1914.

23. Zhao, Z.; Wang, W.; Guo, H.; Zhou, D. Antidepressant-like effect of liquiritin from Glycyrrhiza uralensis in chronic variable stress induced depression model rats. Behav. Brain Res. 2008, 194, 108-113.

24. Kaur, P.; Kaur, S.; Kumar, N.; Singh, B.; Kumar, S. Evaluation of antigenotoxic activity of isoliquiritin apioside from Glycyrrhiza glabra L. Toxicol. In Vitro 2009, 23, 680-686.

25. He, J.; Liao, M.L.; Liu, C.X.; Hao, Z.B.; Zhang T.J. Effect of compatibility on content of amygdalin in Sanao Decoction. Zhongchengyao 2011, 33, 118-120.

26. Yang, C.; Liang, G.Y.; Zhou, J.Y.; Xu, B.X.; Cai, L. Determination of amygdalin in different decoctions of sanaotang by HPLC. Zhongcaoyao 2007, 38, 848-850.

27. Song, W. HPLC determination of ephedrine in the sanaotang preparation. Guizhou Shifan Daxue Xuebao 2011, 29, 138-140. 
28. Yang, C.; Liang, G.Y.; He, Z.Y.; Cao, P.X.; Tian, W.Y.; Cai, L. Determination of glycyrrhizic acid in different decoctions of sanaotang by HPLC and comparison with antifugal effects in vitro. Zhongguo Zhongyao Zazhi 2007, 32, 1031-1034.

29. Yang, C; Liang, G.Y.; Cao, P.X.; He, Z.Y. Determination of ephedrine, glycyrrhizic acid and amygdalin in different decoctions of sanaotang by HPLC. Zhongcaoyao 2008, 39, 372-375.

30. He, J.; Liao, M.L.; Hao, Z.B.; Zhang, T.J. Effects of compatibility on contents of five constituents and finger print in "San-Ao Decoction". Shizhen Guoyi Guoyao 2011, 22, 86-87.

31. Yu, S.; Chen, Y.W.; Zhang, L.; Shan, M.Q.; Tang, Y.P.; Ding, A.W. Quantitative comparative analysis of the bio-active and toxic constituents of leaves and spikes of Schizonepeta tenuifolia at different harvesting times. Int. J. Mol. Sci. 2011, 12, 6635-6644.

32. Li, W.X.; Guo, J.M.; Tang, Y.P.; Wang, H.; Huang, M.Y.; Ding, A.W.; Duan, J.A. Pharmacokinetic comparison of ferulic acid in normal and blood deficiency rats after oral administration of Angelica sinensis, Ligusticum chuanxiong and their combination. Int. J. Mol. Sci. 2012, 13, 3583-3597.

33. Yao, X.; Shang, E.X.; Zhou, G.S.; Tang, Y.P.; Guo, S.; Su, S.L.; Jin, C.; Qian, D.W.; Qin, Y.; Duan, J.A. Comparative characterization of total flavonol glycosides and terpene lactones at different ages, from different cultivation sources and genders of Ginkgo biloba leaves. Int. J. Mol. Sci. 2011, 13, 10305-10315.

34. Tang, Y.P.; Jiang, W.; Wu, Q.C.; Yu, L.; Zhang, L.; Tao, W.W.; Ding, A.W.; You, F.Q.; Duan, J.A. Comparative characteristic of the inflammatory diterpenes in the roots of Euphorbia fischeriana with different preparation method using HPLC-ELSD. Fitoterapia 2012, 83, 427-433.

35. Lv, G.P.; Zhao, J.; Duan, J.A.; Tang, Y.P.; Li, S.P. Comparison of sterols and fatty acids in two species of Ganoderma. Chem. Cent. J. 2012, 6, 1-8.

36. Xie, J.; Zhao, J.; Hu, D.J.; Duan, J.A.; Tang, Y.P.; Li, S.P. Comparison of polysaccharides from two species of Ganoderma. Molecules 2012, 17, 740-752.

37. Tang, Y.P.; Zhu, M.; Yu, S.; Hua, Y.Q.; Duan, J.A.; Su, S.L.; Zhang, X.; Lu, Y.; Ding, A.W. Identification and comparative quantification of bio-active phthalides in essential oils from Si-Wu-Tang, Fo-Shou-San, Radix Angelica and Rhizoma Chuanxiong. Molecules 2010, 15, 341-351.

Sample Availability: Samples of ephedrine, amygdalin, liquiritin, benzoic acid, isoliquiritin, formononetin and glycyrrhizic acid are available from the authors.

(C) 2012 by the authors; licensee MDPI, Basel, Switzerland. This article is an open access article distributed under the terms and conditions of the Creative Commons Attribution license (http://creativecommons.org/licenses/by/3.0/). 